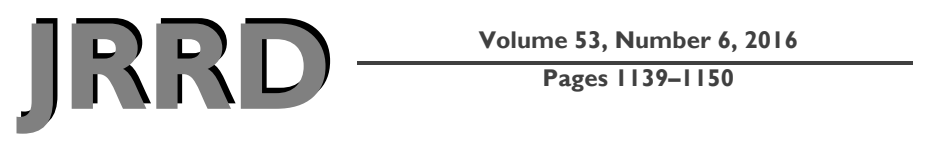

\title{
The perceived functional benefit of dynamic arm supports in daily life
}

\author{
Loek van der Heide, MSc; ${ }^{*}$ Luc de Witte, PhD, MD \\ Research Centre for Technology in Care (CAPHRI), Zuyd University of Applied Sciences, Heerlen, the Netherlands; \\ and School for Public Health and Primary Care, Maastricht University, Maastricht, the Netherlands
}

\begin{abstract}
Dynamic arm supports are provided to assist with activities of daily living (ADLs) in people with limited upperlimb function. However, the perceived functional benefit of these devices in daily life is unknown. Insight into the functional benefit may give direction to the development of new devices and may affect the factors that patients and their health care providers consider during the selection process of a device. A cross-sectional study involving 23 Dutch experienced dynamic arm support users was performed in the Netherlands. The study included a questionnaire, an interview, and an observation of ADL task performance in the domestic setting. Twenty participants completed this study. Five users had a large perceived functional benefit, nine a moderate benefit, and five no benefit (one was unable to indicate the benefit). People with limited functional abilities benefited most. Participants varied in the amount of device use, activities the device is used for, and reasons for using (or not using) the device. On an individual level, selection using a selection tool and a good evaluation of the prescription could improve user-device matches.
\end{abstract}

Key words: activities of daily living, assistive technology, dynamic arm supports, field trial, functional benefit, neuromuscular disorders, occupational therapy, orthotic devices, rehabilitation, upper limb.

\section{INTRODUCTION}

For people suffering from diseases, such as Duchenne muscular dystrophy or multiple sclerosis, or conditions such as stroke, independent eating and teeth brushing are a challenge [1]. Because of limitations in their arm function, these people might rely on the help of caregivers to accomplish activities of daily living (ADLs) and might experience a great loss of independence and control. Dynamic arm supports are a class of assistive technology that could be used to support ADLs for people with limited upper-limb function [2].

A review of the effects and effectiveness of dynamic arm support showed that these devices enable users in controlled conditions to perform tasks they otherwise could not perform independently. However, a relatively high level of nonuse was reported in field studies investigating longitudinal use [3]. For devices to be used in daily life, users must perceive that the benefits outweigh the side effects [4]. For example, Rahman et al. studied the introduction of a dynamic arm support, the WREX (JAECO Orthopedic; Hot Springs, Arkansas), in 17 users. All users showed improved ADL performance, but only four found the device indispensible and continued to use it [5]. Those who discontinued use found, among other reasons, that the WREX interfered with their joystick, armrest, or clothes or that the device was inconvenient because it was attached to the wheelchair while they moved from chair to bed to wheelchair [5]. Haumont et al. found that for users to continue device use, it was

Abbreviations: ADL = activity of daily living, VAS = visual analog scale.

*Address all correspondence to Loek van der Heide, MSc; Zuyd University of Applied Sciences - Research Centre for Technology in Care, Henri Dunantstraat 26419 PB Heerlen, the Netherlands; 003-188-027-2120.

Email: Loek.vanderheide@zuyd.nl

http://dx.doi.org/10.1682/JRRD.2015.06.0099 
necessary that important activities could be performed with increased ease or in less time [6]. Factors negatively affecting use in daily life include not wanting to use the device in the classroom (for student-aged users), the increased need for space, or sufficient functionality without the arm support [5,7]. It is assumed that a perceived functional benefit is essential for devices to make a difference in the daily lives of their users.

At the moment, about 10 different types of dynamic arm supports are commercially available (provided by four suppliers) in the Netherlands. In practice, a specific device is selected by the end-user in close cooperation with a supplier and/or an occupational therapist. During this selection process, attention is paid to the patient's disease (progressive or not), needs and abilities, and environment, among other factors. Subsequently, the user receives the device and can use it for various ADLs at home. A nonoptimal user-device match implies the absence of a perceived functional benefit of the device in daily life. This could mean that another solution would have been better for an individual patient. However, the absence of perceived functional benefit for a group of users could imply that no suitable dynamic arm support is available on the market, which could be a target for future developments. Knowing who does and does not perceive benefits from their arm support could reveal factors that are important to consider during the selection process and provide direction to the development of new devices.

However, there is no insight yet into the perceived functional benefit of these devices in daily life. Therefore, the aim of this study was to assess the perceived functional benefit of dynamic arm supports in experienced users in the Netherlands and to identify the factors that contribute to this perceived functional benefit.

\section{METHODS}

The design was a cross-sectional study among experienced dynamic arm support users. The study consisted of a questionnaire, an interview, and an observation of task performance. Participants, aged 12 yr or older, were provided with a generic (not task-specific) dynamic arm support that allows the arm to be moved vertically (either passive or active), to support in ADLs (not to be used solely at work) between January 2012 and July 2013 [2]. In the Netherlands, four companies provide dynamic arm supports. Participants were invited to participate by the supplier from whom they received their dynamic arm support. The four suppliers sent an information letter, an application form, and an informed consent form by mail to everybody who met the criteria. The suppliers contacted via email or phone those who did not apply for the study after a month and asked them whether they would like to participate. In order to minimize selection bias, participants were explicitly told in the information letter that this study was also directed at nonusers.

Participants received the questionnaire by mail or email. People who filled in and returned the questionnaire were contacted by phone by the researcher and an appointment was made for the interview/observation. The interview and observation were performed at the participant's home and were conducted by an interviewer and an observer (both occupational therapists). Two interviewers and two observers conducted all home visits in fixed pairs. The pairs were randomly assigned to the participants.

\section{Questionnaire}

Participants filled in the questionnaire either online or on paper at home. The goal of the questionnaire was to assess how ADLs are usually performed, whether this takes a lot of effort, and whether the dynamic arm support is used. The long version of the Assessment of Life Habits questionnaire (English, 3.0) was adapted to form the basis of our questionnaire [8]. Only those activities in which the upper limb is involved and that could be performed in a wheelchair were selected. This resulted in a list of 51 items. These items were translated into Dutch independently by two researchers. We discussed and came to a consensus on differences in translation and formulation of the questions. Additionally, we added an item to assess the use or nonuse of the dynamic arm support during specific activities. Applicability of the questionnaire was assessed in pilot interviews. Furthermore, participants were asked to fill in participant characteristics, such as age, condition, type of dynamic arm support, and mounting of the device (to a table, wheelchair, tripod, etc.).

\section{Interview}

The interview started with assessment of the perceived functional benefit by asking the following two questions: "How would you rate your ability to perform activities of daily living with your dynamic arm support?" and "Imagine you do not have your dynamic arm 
support. How would you rate your ability to perform activities of daily living?” Both questions were rated on a visual analog scale (VAS) from 0 to 100. The difference between the two scores represents the perceived functional benefit. A score of $\leq 0$ (score with device - score without device) represents no perceived functional benefit, a score between 0 and 50 was considered a moderate perceived functional benefit, and a score of $\geq 50$ was considered a large perceived functional benefit.

Based on the questionnaire responses, we developed an interview scheme to assess why people use or do not use their arm support in performing ADLs. If participants indicated that an activity took a lot of effort to accomplish while not using the dynamic arm support, was performed by others, or was not performed, they were asked why the device was not used. If participants indicated that a specific activity required a lot of effort while their dynamic arm support was used, they were asked why performance of that activity still required a lot of effort. Additionally, if the arm support was used for an activity, participants were asked for the main reason they were unable to perform the task without dynamic arm support. Furthermore, questions were asked to assess frequency of use and satisfaction with the device.

\section{Observation of Task Performance}

The quality of ADL task performance was assessed in the home environment for six tasks. These tasks were (1) opening and closing a door and shaking hands, (2) drinking from a glass, (3) using a keyboard, (4) combing hair, (5) putting on a coat, and (6) reaching for a book on a coffee table. These activities were selected because they represent tasks that most people have to perform in some way in daily life, they are regarded as important, and they originate from different ADL domains [1,8]. Each ADL task was divided into subtasks by occupational therapists. The subtask performance was scored on a scale from 0 to 4 , ranging from competent (4) to unable to perform because of functional limitations (0). This scoring procedure was based on the Assessment of Motor and Process Skills scoring procedure [9]. In addition, the occupational therapists noted indicators of movement quality, which consisted of compensatory movements, double/single handed, reasons for not accomplishing tasks, use of other assistive devices, speed of movement, help required, and effort. The observers were trained to use the scoring procedure to diminish the interrater variability in scores appointed.
Participants were, if possible, asked to perform each of the six ADL tasks with and without their device. They were instructed to perform the task as naturally as possible. If they normally used other assistive devices, such as adapted spoons, they were allowed to use these. They were also encouraged to perform activities they normally do not perform. The Brooke scale was applied to provide an overall measure of upper-limb function [10]. The score was assessed for the right and the left arm separately.

\section{Data Analysis}

Answers on the interview questions were labeled. The scores representing the quality of ADL performance with and without dynamic arm support were compared for each subtask for each participant. The data derived from the observation were converted into categories: (1) activity was performed better with device, (2) activity was performed better without device, or (3) performance with and without device was equal. Changes were not determined when tasks could not be performed because of the fixed location of the arm support or because required objects were unavailable in participants' homes.

Participant characteristics were described for each case. Descriptive statistics (mean \pm standard deviation, frequencies) were calculated for age and the quality of subtask performance for the three groups of participants who differed in the perceived functional benefit (large, moderate, none). Subsequently, these groups were qualitatively compared for personal characteristics, the quality of ADL task performance, and the (reasons for) use (and nonuse) of the dynamic arm support in daily life.

\section{RESULTS}

Of 153 people invited to participate in this study, 29 applied for the study and received the questionnaire, and 23 people filled in the questionnaire. A total of 20 people were visited by the researchers for the interview and observation (Figure 1).

Age of the participants ranged from 14 to $75 \mathrm{yr}$ (mean $54.8 \pm 14.6$ yr). Five participants were diagnosed with spinal muscular atrophy, and other diagnoses were, among others, spinal cord injury, multiple sclerosis, and amyotrophic lateral sclerosis (Table 1). Participants received various types of dynamic arm supports (Figure 2) ranging from passively to actively actuated devices from Armon Products (Rotterdam, the Netherlands) and several 


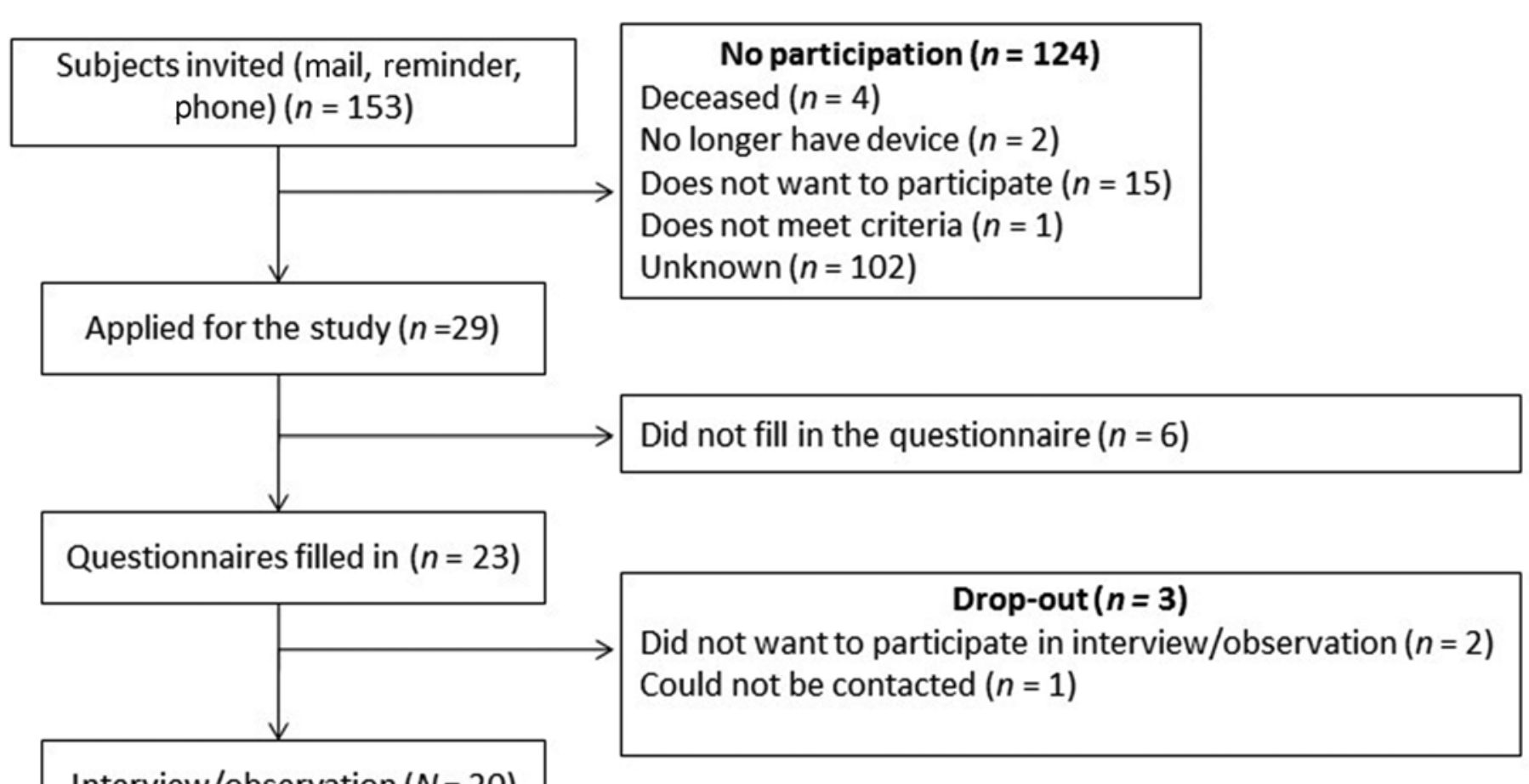

Figure 1.

Participant flowchart.

products developed by Focal Meditech (Tilburg, the Netherlands) [2]. Arm function assessed with the Brooke scale varied from the highest to the lowest possible score: from optimal arm function "starting with the hands at the sides, the patient can abduct the arms in a full circle until they touch above the head" (score 1) to very little arm function, in which the user "cannot raise hands to the mouth and has no useful function of the hands" (score 6) [10] (Table 1). Use of the dynamic arm support in daily life varied between "never" $(n=4)$ and continuously $(n=$ 6). Participants gave two reasons for nonuse: deterioration of the disease $(n=3)$ and an incorrectly adjusted device $(n=1)$.

The perceived functional benefit was assessed for 19 participants during the interview, because one participant found it too difficult to answer the required questions. The extent to which dynamic arm supports were perceived to increase the ability to perform ADLs-the perceived functional benefit-differed widely among participants (Figure 3). Five participants reported that the functional abilities did not increase with the dynamic arm support, meaning that there was no perceived functional benefit (one of them had a negative perceived functional benefit). In nine participants there was a moderate perceived functional benefit. Five participants reported a relatively large improvement in the ability to perform ADLs with dynamic arm support ( $\geq 50$ points). These participants had a large perceived functional benefit of their device. Overall, people with a large functional benefit tended to have a limited perceived ability to perform ADLs without a device. People that did not have a benefit indicated that, without the device, they were already able to perform ADLs quite well (Figure 3).

Overall, the frequency of use was related to the perceived functional benefit (Figure 4). Participants who perceived a benefit used their device continuously, whereas participants who did not perceive a benefit were more likely to never use the device in daily life. An exception was one participant who, because of functional deterioration, did not use the arm support anymore, but had used the device about $17 \mathrm{~h} / \mathrm{d}$ in the past. Some participants were completely wheelchair confined, whereas other participants could still walk and never used a wheelchair in daily life. In most cases, the dynamic arm support was attached to the wheelchair, but when somebody was not wheelchair bound, the arm support was attached to a tripod, 
Table 1.

User characteristics.

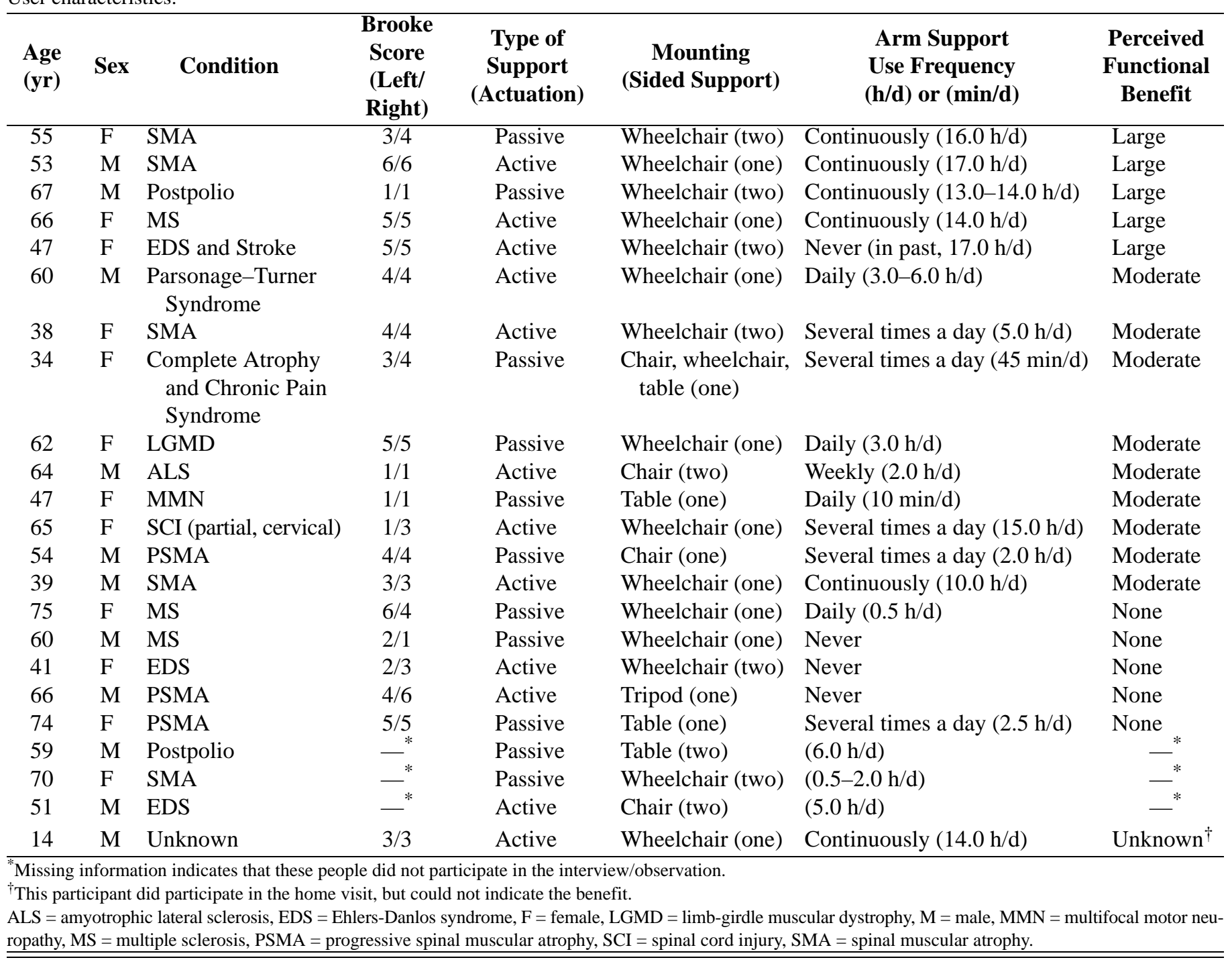

a normal chair, or a table. All participants with a large perceived functional benefit had their device attached to a wheelchair (Table 1).

Participants were asked to indicate for which ADLs they used their dynamic arm support on a normal day. Some participants used their dynamic arm support for one or two specific tasks, such as eating, cooking, or desktop tasks (using the computer, writing, reading, phoning). Others used it for many tasks covering different domains and activities happening at different locations in the house [8]. The majority of the participants $(\geq 10)$ used the device in order to eat, drink, use a computer, and use the phone (Figure 5).

\section{Reasons for Using the Dynamic Arm Support in Daily Life}

We asked participants for the main reason they could not perform specific activities without dynamic arm support. This provides insight into the reasons, which vary widely between participants, for using the device in daily life. However, overall, users indicated that devices are used when they reduce tremors, save energy, reduce pain, provide stability, and/or assist with tasks that require less effort/strength to perform. Additionally, users indicated that without support it is impossible to lift objects, such as a telephone or food on a spoon; to perform activities for a longer period of time; and to perform repetitive movements (e.g., eating, brushing teeth). 


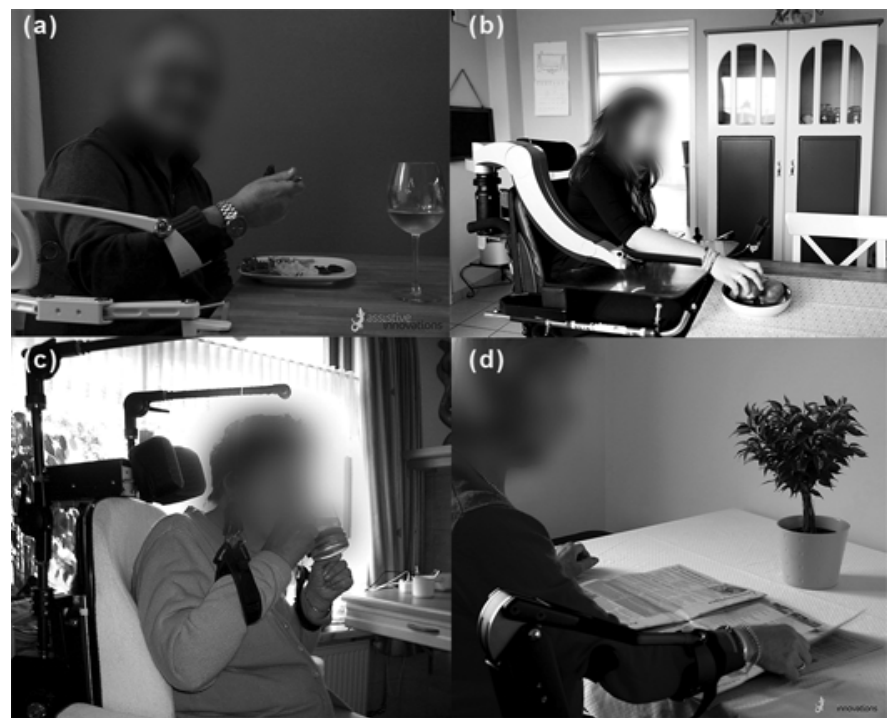

Figure 2.

Photographs of some devices used by participants. Photos (a) Armon Edero and (d) Armon Ayura are printed with permission from Assistive Innovations (Didam, the Netherlands). Pictures (b) Darwing and (c) Balancer are printed with permission from Focal Meditech (Tilburg, the Netherlands).

\section{Reasons for Not Using the Dynamic Arm Support in Daily Life}

When asked for reasons for not using the arm support for specific tasks, participants indicated that it may be inconvenient to perform certain tasks with dynamic arm support. Examples include getting into the bathroom with wheelchair and dynamic arm support and using the device in the bathroom, because there is limited space to move around. In some cases the device does not support the required movements sufficiently; for example, it can be impossible to reach for a sink or a shelf. A risk of fumbling deters some people from transporting items, such as drinks or food. Additionally, users argued that they prefer to spend the limited amount of energy they have on tasks they enjoy. As a consequence, household chores and selfcare activities (getting dressed, showering) were often performed by caregivers. Often other solutions were sought in order to be able to perform tasks independently; for example, an e-reader could be used instead of a book and ADL support dogs could offer help in daily life. Some dynamic arm supports were attached to a table or another fixed location, which prevented people from using the device in other places. Users who could per-

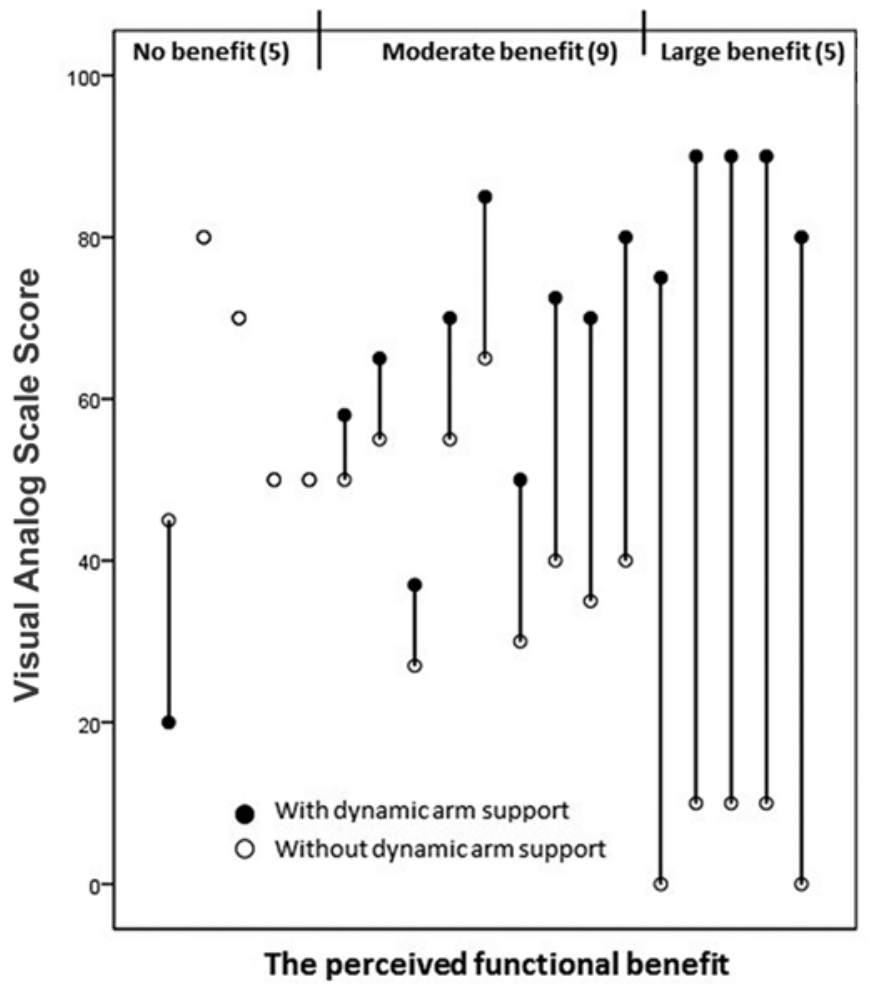

Figure 3.

The perceived functional benefit of individual users. The length of the bars represents the perceived functional benefit. If only one hollow dot is shown, the ability to perform activities of daily living is equal with and without arm support.

form some tasks themselves and did not need the dynamic arm support continuously said that it takes too much effort to get the device, put their arm in it, and use it for a specific task. Other reasons for not choosing to use a dynamic arm support were a severely decreased arm/hand function to the extent that help from others is required, that a device does not improve the ability to perform tasks, and that a device does not suppress tremors.

\section{Reasons Activities Require Effort Although the Dynamic Arm Support Is Used}

Activities with arm support still require effort because of decreased coordination and strength, levels of pain and fatigue, a lack of energy, and incorrectly adjusted devices. Eleven participants used their device for drinking; however, the activity was still performed with difficulty by four, because it was difficult for them to hold the cup and bring it to the mouth. 


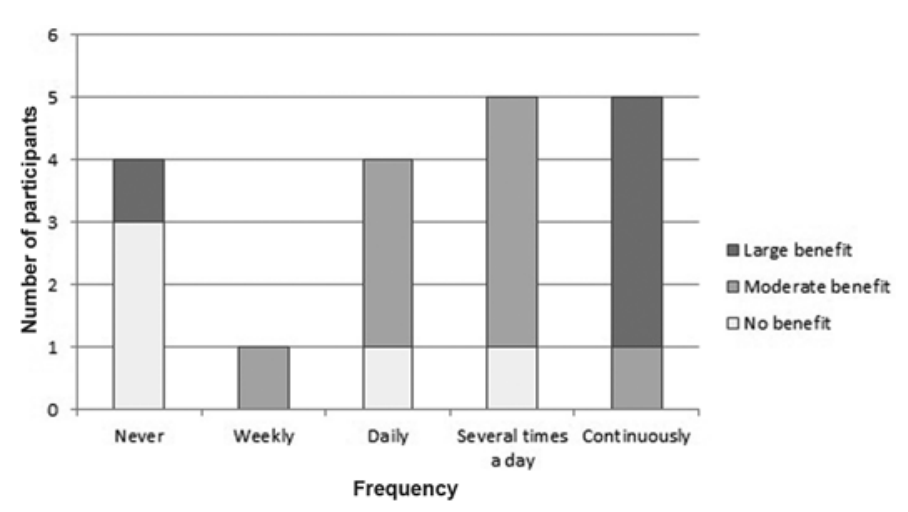

Figure 4.

Frequency of use and perceived functional benefit.

\section{Observation of Activity of Daily Living Task Performance}

Task performance of 6 tasks, divided into 29 subtasks, was assessed with and without dynamic arm support in the participants' home environment. Overall, people with a large perceived functional benefit showed an improved quality of performance in many (on average 14 of the 29) subtasks (Figure 6). Subtasks that involved lifting objects toward and above shoulder level (bringing a glass toward the mouth and combing hair) also improved with dynamic arm support in people without a perceived functional benefit (Table 2). These tasks require less effort or are performed more efficiently, or people use less compensatory movements. However, in people who do not perceive a benefit, subtasks were often performed better without dynamic arm support (on average 8.6 of the 29 subtasks) or the observed quality was similar (on average 9.6 of the 29 subtasks) (Figure 6 and Table 3).

The observed quality of ADL performance reveals that people with a large functional benefit were often unable to perform tasks without dynamic arm support at all or showed a very low ability to perform tasks (Figure 7). People with a moderate or no perceived functional benefit were, without arm support, already able to perform many movement components (>40\%) without problems (score 4).

\section{DISCUSSION}

The aim of this study was to assess the perceived functional benefit of dynamic arm supports in daily life and identify factors that contribute to this perceived benefit. The extent to which dynamic arm supports contributed to the perceived ability to perform ADLs varied widely among the participants. However, for devices to be used in daily life it is essential that the user perceives the device as functionally beneficial. This has also been reported for assistive technology in general [11].

Five out of the 19 participants did have a large perceived functional benefit of the arm support. These devices were frequently used, and in these cases it is assumed that the user-device match was good. Five (out of 19) participants did not perceive a functional benefit of their device. These participants were already relatively functional in daily life without a device. For those with a moderate benefit (9 out of 19), there was a minor improvement in the perceived ability to perform ADLs. Additionally, the perceived ability to perform ADLs with dynamic arm support was given a score between 37 and 85 points (on a VAS scale $0-100$ ). Based on these outcomes, there seems to be further room for dynamic arm supports to increase the ability to perform ADLs with a device. This leads to the suggestion that the match between user and dynamic arm support is not fully optimal in each case. A nonoptimal match can be (1) the result of an incorrectly chosen device (another type of dynamic arm support or other solution would have fitted better with this user), (2) because there is nothing available on the Dutch market that matched better, or (3) because the match was optimal at the moment of selection but not at the time of this study. Based on the findings of this study, it is impossible to determine the appropriate reason for each individual user. However, several factors were found related to perceived functional benefit.

It appeared that people who were already able to perform ADLs quite well often did not benefit (sufficiently) from their dynamic arm support. Various types of dynamic arm supports with different amounts of support provided and two expected target groups (passive, active) were involved in this study. These findings could therefore suggest that no suitable devices seem to be available for people who are already relatively functional but need support in antigravity movements. Because the benefit of the device has to outweigh the side effects, it would probably be worth the effort to develop a new dynamic arm support, possibly focusing on limiting the side effects, for this target group. It is unknown whether users expected an increase in functional ability when they selected and received their device. It is important to assess the patient's current functional ability in daily life and the potential gain with the device; in the future, these considerations could influence the decision of whether it would be wise 
JRRD, Volume 53, Number 6, 2016

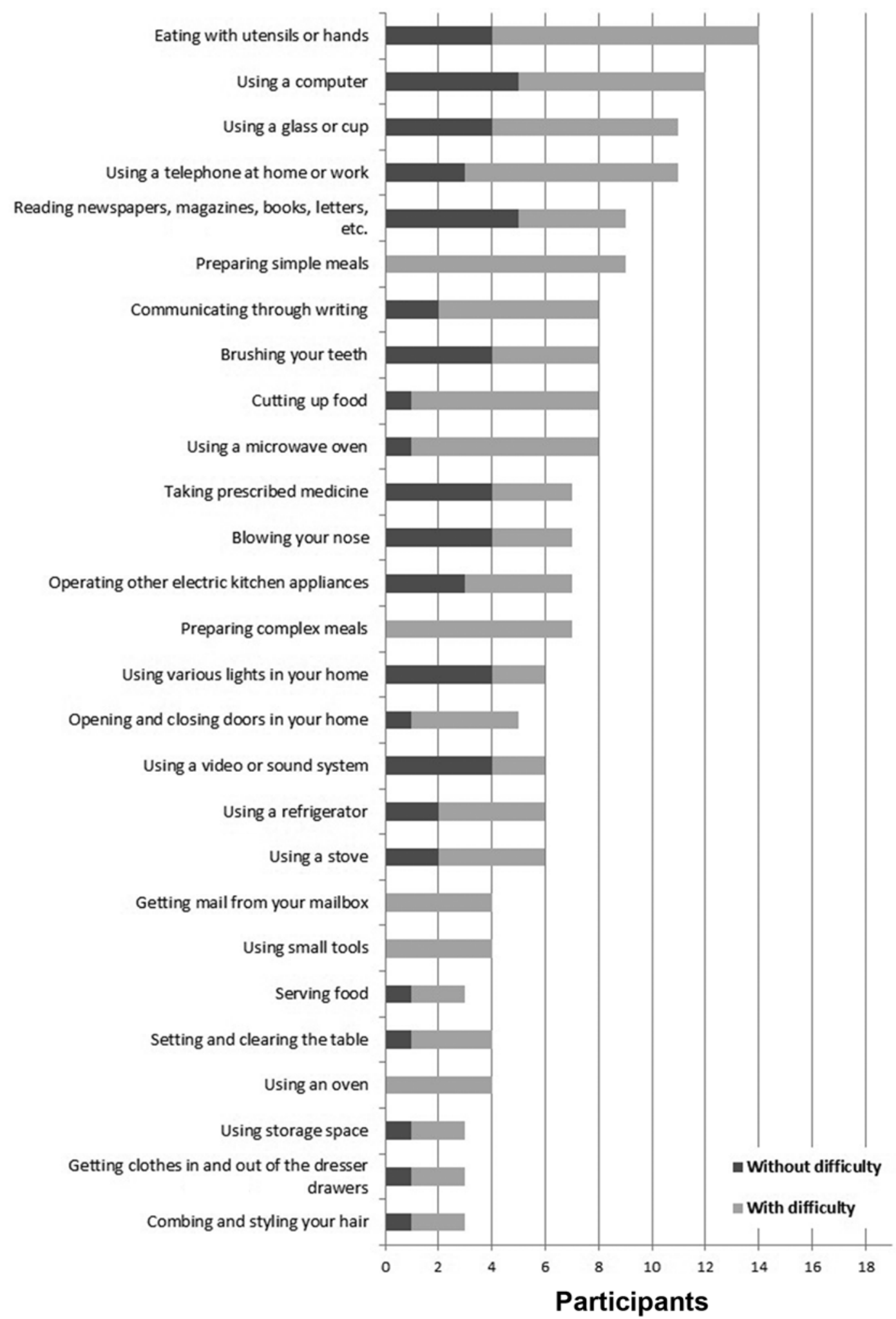

Figure 5.

Use of the dynamic arm support during activities of daily living (ADLs) (for the 19 participants who use the device in daily life) and the perceived difficulty of ADL task performance with the dynamic arm support. 


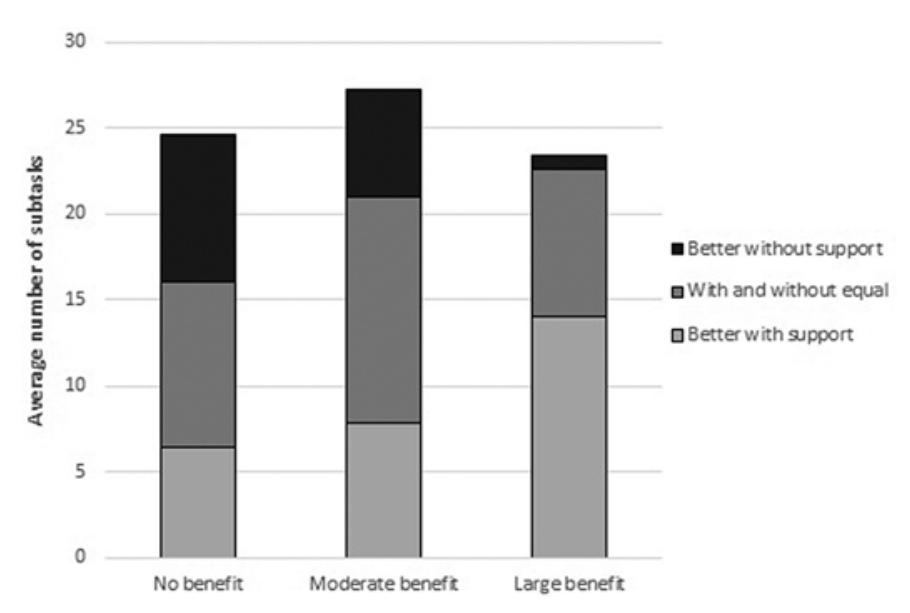

Figure 6.

Observed quality of task performance with use of dynamic arm support by perceived functional benefit. (See Table $\mathbf{3}$ for numerical representation.)

to opt for an arm support at all. The assessment of ADL tasks used in this study might play a role in this respect.

Arm function of users who benefited largely from passively actuated devices varied between Brooke score 1 and 4. Arm function of users who benefited largely from actively actuated devices varied between Brooke score 5 and 6 . This is roughly in line with existing ideas about selecting actively actuated devices for people with a severely decreased arm function [12]. Although the majority of the participants had a device (passive or active) that corresponded with their Brooke score, many did not benefit sufficiently from their device. The arm function should be taken into account during the selection of devices, but it is insufficient to select either a passive or an active device based on arm function alone.

All participants with a large perceived functional benefit had a wheelchair-bound arm support. Users with an arm support attached to a chair (with wheels for foot propulsion), table, or tripod perceived their arm support as less beneficial. These participants often indicated that they did not use the device because activities must be performed at a certain location. It is therefore questionable whether attachment of the arm support to a chair, table, or tripod is a good solution. During the selection process, it could be wise to also investigate dedicated devices, such as eating and drinking devices and page turners. On the other hand, this small benefit implies that, at this moment, there is no suitable solution available on the Dutch market for the group of users who have problems in performing ADLs because of decreased arm function but who are not wheelchair dependent. In the

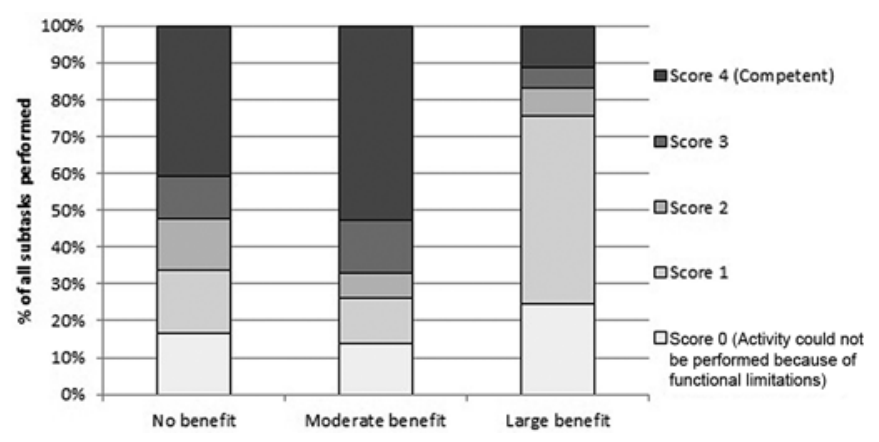

Figure 7.

Observed quality of task performance without use of dynamic arm support by perceived functional benefit. Score $4=$ competent. Score $3=$ the observer questions whether or not there was a problem. Score 2 = some increased effort or inefficiency noted could be slight or more pronounced. Score 1 = an unacceptable amount of effort or inefficiency, imminent safety risk, or need for assistance was noted. Score $0=$ unable to perform because of functional limitations.

United States, body-worn dynamic arm supports are available [13]. The potential for this kind of device in the Netherlands should be investigated as well by manufacturers/developers.

At the time of this study, participants had possessed their dynamic arm supports for a period ranging from 1.0 to $2.5 \mathrm{yr}$. Some participants without a perceived benefit during the study could have had a perceived benefit at the time of selection. This was not assessed in this study; however, during the interviews three participants mentioned that they did not use the arm support anymore because of functional deterioration. This implies that problematic ADLs for which the arm support was prescribed are probably not supported anymore. Additionally, nonuse of these devices can be considered an inefficient use of resources. It therefore is important that each dynamic arm support prescribed is followed by an evaluation after a certain period of time, at which point other solutions can be sought to support independence again if the benefit has deteriorated or is absent.

We found individual differences in how dynamic arm supports were used in daily life and why people chose to use or not to use the device. Each user considered whether it would be beneficial to use an arm support for specific daily activities. Users took into account what activities they find important to do independently in daily life, how much energy they have, environmental facilitators and barriers, whether activities can be performed in other ways (without support/adaptations), and so on. During 
Table 2.

Observed quality of activity of daily living (ADL) performance with and without dynamic arm support compared $(N=19)$.

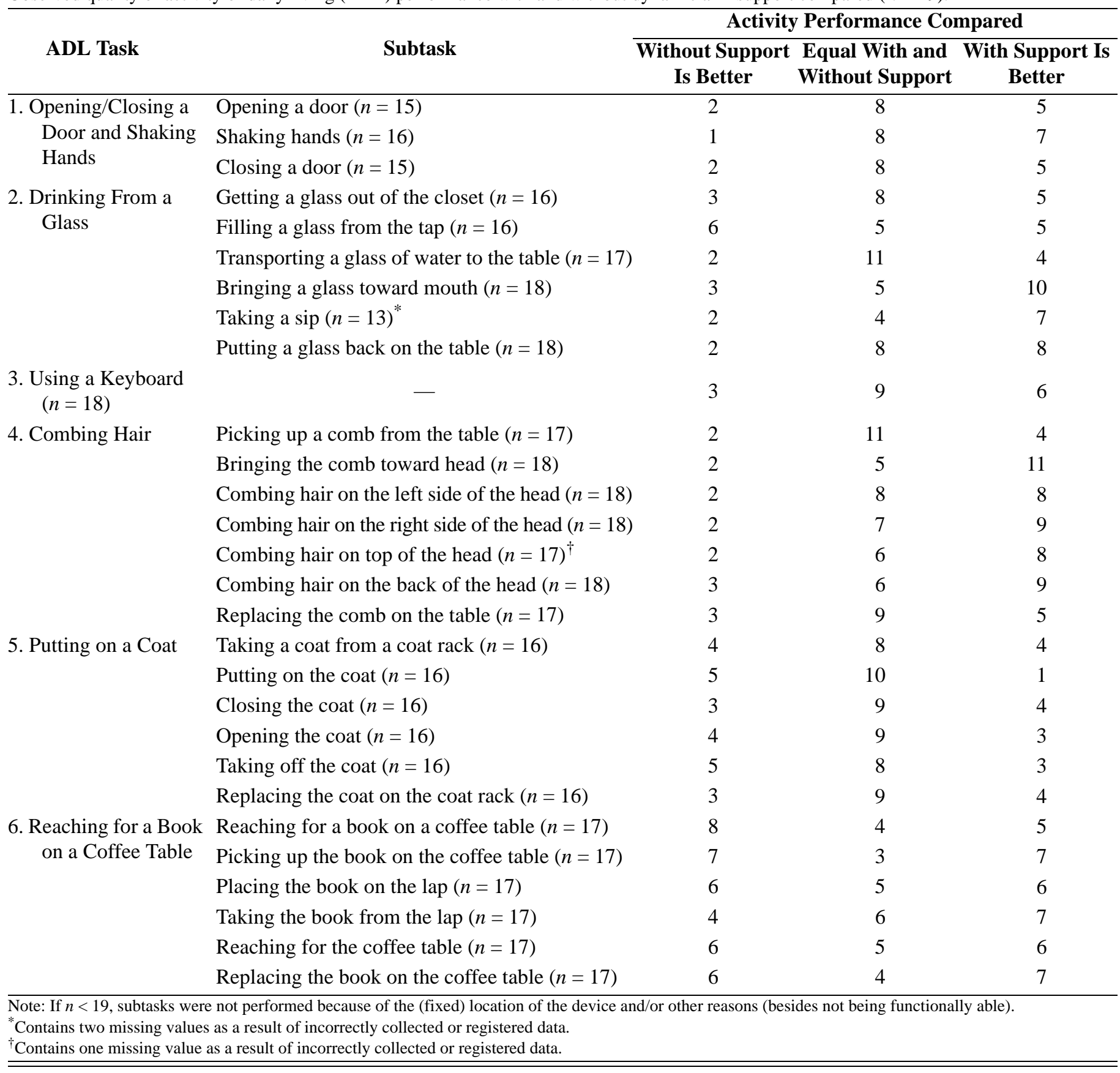

Table 3.

Observed quality of task performance with use of dynamic arm support by perceived functional benefit. (See Figure 6 for visual representation.)

\begin{tabular}{lccc}
\hline Quality of Task Performance & No Benefit, Mean (SD) & Moderate Benefit, Mean (SD) & Large Benefit, Mean (SD) \\
\hline Better with Support & $6.4(5.9)$ & $7.9(8.0)$ & $14(11.2)$ \\
With and Without Equal & $9.6(11.6)$ & $13.1(5.9)$ & $8.6(8.3)$ \\
Better Without Support & $8.6(11.8)$ & $6.2(8.1)$ & $0.8(1.8)$ \\
\hline
\end{tabular}

$\mathrm{SD}=$ standard deviation. 
the selection process, people involved (professionals and their clients) have to anticipate these individual differences. Additionally, the various reasons participants gave for using the device in daily life, such as to reduce tremors during ADLs, prevent fatigue, and compensate for the lack of muscle force, indicate that it would be helpful to focus on the functional limitations as a consequence of the disease rather than the diagnosis itself.

A choice for a specific device has to be made based on the user's needs and wishes. It therefore is essential that the user is actively involved in the selection process. Development of a selection tool will support involving the user in this process by calling attention to all important aspects. This will help in finding the best solution available for each person with limited arm function. Methods used in this study could be incorporated in the selection tool if the predictive capacity of these methods, with respect to the perceived functional benefit, is clear. A challenge will be to identify how the factors that contribute to a perceived benefit predict the benefit of a specific device in the future. Additionally, it was difficult to find out why some people benefit from their device while others do not. The perceived functional benefit is affected by many different aspects, which also differ between individuals. Additionally, besides the aspects assessed in this study, other factors, for example, related to the Matching Person and Technology Model [14], may also affect the perceived functional benefit. This should be kept in mind during the development of a selection tool.

\section{Methodological Considerations}

This study has some methodological considerations. First, only 15 percent of all persons invited for the study participated. As a consequence, selection bias could have occurred if people who use their device and perceive the arm support as beneficial were more likely to apply for the study than people who did not use the device often in daily life. As a consequence, the level of nonuse (17.4\%) might be an underestimation of the actual nonuse. This would, however, underline the importance of improving user-device match even more. Second, some data were missing in the interview, because for some participants the interview was very long. However, it is expected that the interview as a whole provides a valid overview of the variety of answers on the posed questions. Third, a difference of at least 50 points between the ability to perform ADLs with and without a device was regarded as a large perceived functional benefit. Theoretically, it is impossible to have a large benefit if the ability to perform tasks without device already exceeds the score of 50 (VAS scale). The two questions determining the functional benefit are subjective questions, implying that the boundaries on the VAS scale are relative. Additionally, the ceiling of the VAS scale was never reached. Based on this, it is assumed that the defined categories for the perceived functional benefit are meaningful. Fourth, it could be the case that the perceived functional benefit and observed quality of task performance are overestimated in people with a large perceived benefit. People who used the arm support often in daily life found that they were very well able to perform tasks with the device (which is also reflected in the observation of task performance) because they were simply used to using the device in daily life. However, people with a large perceived benefit were more often unable to perform ADL tasks without the device at all. So, it is likely that in these cases use of an arm support more easily results in an improvement of ADL performance.

\section{CONCLUSIONS}

The perceived functional benefit varies widely among users but is essential for dynamic arm supports to be used in daily life. People with limited functional abilities benefited most. The user-device match was not optimal in the majority of the cases. Overall, it is a complex task to determine why some people benefit from their device while others do not because reasons for benefit are affected by many user-specific aspects. The individual variation stresses the importance of making the match between user and dynamic arm support on an individual level. More than is done in current practice, the functional abilities, needs, and environment of the potential user should be taken into account. A selection tool could be developed to support patients and their care professionals in choosing the best solution available. Finally, each prescription should be evaluated after a certain period of time by assessing the extent to which the device contributes to the expected ability to perform ADLs.

\section{ACKNOWLEDGMENTS}

\section{Author Contributions:}

Study concept and design: L. van der Heide, L. de Witte. Acquisition of data: L. van der Heide.

Analysis and interpretation of data: L. van der Heide, L. de Witte. Drafting of manuscript: L. van der Heide. 
Critical revision of manuscript for important intellectual content:

L. de Witte.

Final approval of manuscript: L. de Witte.

Financial Disclosures: The authors have declared that no competing interests exist.

Funding/Support: This material was based on work supported by Pieken in de delta, agentschap nl (Award \#PID 102055).

Additional Contributions: The authors would like to thank our colleague Gert Jan Gelderblom for his highly appreciated contribution to this work. The authors would also like to thank Robbert Hendrix, Ruben Joosten, Bjorn Sponnik, and Simone Zelissen for their contribution to this work. Luc de Witte, PhD, MD, is now with Health Services Research, School of Health and Related Research, Centre for Assistive Technology and Connected Healthcare, the University of Sheffield, United Kingdom.

Institutional Review: The study was approved by the Medical Ethical Committee of Atrium-Orbis-Zuyd (Heerlen, The Netherlands). All participants signed informed consent forms.

Participant Follow-Up: The authors plan on informing the participants of the publication of this study via the companies who delivered the dynamic arm supports involved in this study.

\section{REFERENCES}

1. Janssen MM, Bergsma A, Geurts AC, de Groot IJ. Patterns of decline in upper limb function of boys and men with DMD: An international survey. J Neurol. 2014;261(7): 1269-88. Erratum in: J Neurol. 2014;261(7):1289-90. [PMID:24687893] http://dx.doi.org/10.1007/s00415-014-7316-9

2. Van der Heide LA, van Ninhuijs B, Bergsma A, Gelderblom GJ, van der Pijl DJ, de Witte LP. An overview and categorization of dynamic arm supports for people with decreased arm function. Prosthet Orthot Int. 2014;38(4): 287-302. [PMID:23950551] http://dx.doi.org/10.1177/0309364613498538

3. van der Heide LA, Gelderblom GJ, de Witte LP. Effects and effectiveness of dynamic arm supports: A technical review. Am J Phys Med Rehabil. 2015;94(1):44-62. [PMID:24901759] http://dx.doi.org/10.1097/PHM.0000000000000107

4. Rahman T, Sample W, Jayakumar S, King MM, Wee JY, Seliktar R, Alexander M, Scavina M, Clark A. Passive exoskeletons for assisting limb movement. J Rehabil Res Dev. 2006;43(5):583-90. [PMID:17123200] http://dx.doi.org/10.1682/JRRD.2005.04.0070

5. Rahman T, Sample W, Seliktar R, Scavina MT, Clark AL, Moran K, Alexander MA. Design and testing of a functional arm orthosis in patients with neuromuscular diseases. IEEE Trans Neural Syst Rehabil Eng. 2007;15(2):244-51. [PMID:17601194] http://dx.doi.org/10.1109/TNSRE.2007.897026
6. Haumont T, Rahman T, Sample W, M King M, Church C, Henley J, Jayakumar S. Wilmington robotic exoskeleton: A novel device to maintain arm improvement in muscular disease. J Pediatr Orthop. 2011;31(5):e44-49.

[PMID:21654447]

http://dx.doi.org/10.1097/BPO.0b013e31821f50b5

7. Kumar A, Phillips MF. Use of powered mobile arm supports by people with neuromuscular conditions. J Rehabil Res Dev. 2013;50(1):61-70. [PMID:23516084] http://dx.doi.org/10.1682/JRRD.2012.03.0047

8. Fougeyrollas P, Noreau L, Bergeron H, Cloutier R, Dion SA, St-Michel G. Social consequences of long term impairments and disabilities: Conceptual approach and assessment of handicap. Int J Rehabil Res. 1998;21(2):127-41. [PMID:9924676] http://dx.doi.org/10.1097/00004356-199806000-00002

9. Fisher AG. Assessment of motor and process skills. Fort Collins (CO): Three Star Press; 2001.

10. Brooke MH, Griggs RC, Mendell JR, Fenichel GM, Shumate JB, Pellegrino RJ. Clinical trial in Duchenne dystrophy. I. The design of the protocol. Muscle Nerve. 1981; 4(3):186-97. [PMID:7017401] http://dx.doi.org/10.1002/mus.880040304

11. Dijcks BP, De Witte LP, Gelderblom GJ, Wessels RD, Soede M. Non-use of assistive technology in The Netherlands: A non-issue? Disabil Rehabil Assist Technol. 2006;1(1-2):97-102. [PMID:19256172] http://dx.doi.org/10.1080/09638280500167548

12. Herder JL, Vrijlandt N, Antonides T, Cloosterman M, Mastenbroek PL. Principle and design of a mobile arm support for people with muscular weakness. J Rehabil Res Dev. 2006;43(5):591-604. [PMID:17123201] http://dx.doi.org/10.1682/JRRD.2006.05.0044

13. Rahman T, Sample W, Seliktar R, Alexander M, Scavina M. A body-powered functional upper limb orthosis. J Rehabil Res Dev. 2000;37(6):675-80. [PMID:11321003]

14. Scherer MJ. Living in the state of stuck. 4th ed. Brookline (MA): Brookline Books; 2005.

Submitted for publication June 1, 2015. Accepted in revised form November 10, 2015.

This article and any supplementary material should be cited as follows:

van der Heide L, de Witte L. The perceived functional benefit of dynamic arm supports in daily life. J Rehabil Res Dev. 2016;53(6):1139-50. http://dx.doi.org/10.1682/JRRD.2015.06.0099

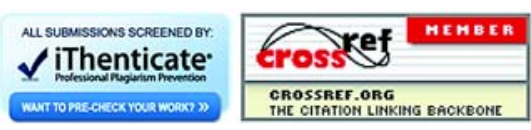

